\title{
Broadband THz Modulators Based on Multilayer Graphene on PVC
}

\author{
Emine Kaya ${ }^{1}$, Nurbek Kakenov ${ }^{2}$, Coskun Kocabas ${ }^{2}$, Hakan Altan ${ }^{3}$, Okan Esenturk ${ }^{1}$ \\ ${ }^{1}$ Department of Chemistry, Middle East Technical University, 06531 Ankara, Turkey \\ ${ }^{2}$ Department of Physics, Bilkent University, 06800 Ankara, Turkey \\ ${ }^{3}$ Department of Physics, Middle East Technical University, 06531 Ankara, Turkey
}

\begin{abstract}
In this study we present the direct terahertz timedomain spectroscopic measurement of CVD-grown multilayer graphene (MLG) on PVC substrate with an electrically tunable Fermi level. In a configuration consisting MLG and injected organic dopant, the transmitted intensity loss of terahertz radiation was observed with an applied voltage between 0 and 3.5 V.We showed that MLG on PVC devices provided approximately $100 \%$ modulation between 0.2 and $1.5 \mathrm{THz}$ at preferentially low operation voltage of ca. $3 \mathrm{~V}$. The observed modulation bandwidth in terahertz frequencies appears to be instrument limited.
\end{abstract}

\section{INTRODUCTION}

$\mathrm{T}$ $\mathrm{Hz}$ modulators are structures capable of actively controlling the amplitude (or phase) of the transmitted (or reflected) $\mathrm{THz}$ waves.[1] Different types of $\mathrm{THz}$ modulators have been intensely investigated such as semiconductor heterostructures containing a two dimensional electron gas (2DEG)[2], metal-oxide-semiconductor technology [3] and metamaterial-based modulators [4]. A room temperature 2DEG modulator was demonstrated by Kleine et al. Although RT operation is practical, poor modulation depths of 3\%-4\% have been reported due to the usage of metal gates. Gao et al. reported 50\% amplitude modulation with gated single-layer graphene using metallic ring apertures. This spatial light modulator can operate with low bias voltage at room temperature but it has a narrow bandwidth. Recent metamaterial based modulators can also have around 50\% modulation. However, their narrowband operational range and polarization-dependent response may limit their future applications. Single layer graphene offers very low insertion loss $(0.2-0.5 \mathrm{~dB})$. Kakenov et al. presented an ionic liquid based $\mathrm{THz}$ intensity modulator.[5] Modulator structure consisted of two graphene coated quartz wafers with a spacer in between which is filled with ionic liquid. Due to efficient mutual gating of graphene electrodes and ionic liquid more than $50 \%$ modulation depth was obtained.

We experimentally demonstrate an excellent performance on $\mathrm{THz}$ modulation based on MLG/ionic liquid structures on Polyvinyl chloride (PVC) substrate. The modulation covers a broadband frequency range from 0.2 to $1.5 \mathrm{THz}$ with the modulation depth of up to $99 \%$ with application of very low voltage of $3 \mathrm{~V}$. This high modulation depth stems from the strong gating effect of ionic liquid. To our knowledge, this is one of the highest modulation depths from graphene based $\mathrm{THz}$ modulators with such a broad $\mathrm{THz}$ range.

\section{RESULTS}

In Figure $1 \mathrm{THz}$ transmission of modulator device is given in a range from 0.2 to $1.5 \mathrm{THz}$ at representative voltages. The observed modulation with the set voltage appears to be independent of the $\mathrm{THz}$ frequency and only instrument limited. Up to $\sim 2 \mathrm{~V}$ less than $20 \%$ modulation is observed. A modulation between 40 and $60 \%$ at ca $2.5 \mathrm{~V}$ can be achieved.

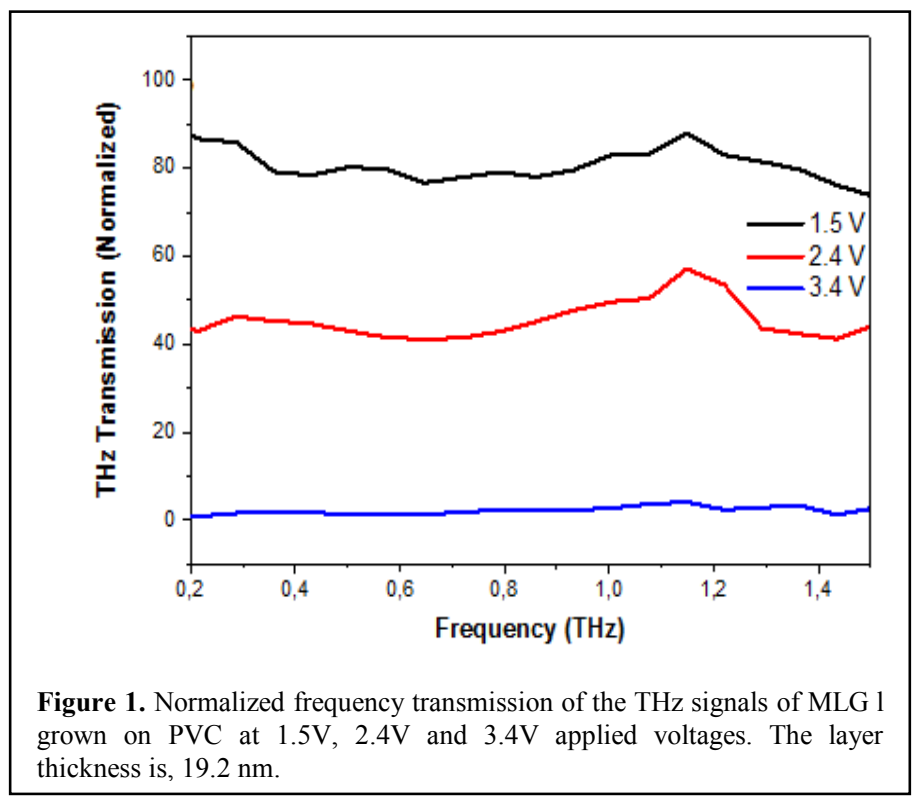

A complete modulation has been achieved with modulator devices at voltages beyond $3 \mathrm{~V}$. As clearly seen from the data presented in the figure, the modulation depth is significantly improved compared to single and multilayer devices.

\section{SUMMARY}

In conclusion, we fabricated $\mathrm{THz}$ modulators using CVDgrown graphene layers doped with ionic liquid electrolyte and investigated $\mathrm{THz}$ transmission by $\mathrm{THz}$ time-domain spectroscopy. High modulation depth over such a broad spectrum and a simple device structure brings significant importance toward application of this type of device in $\mathrm{THz}$ and related technologies.

\section{REFERENCES}

[1] M. Liu, X. Yin, E. Ulin-Avila, B. Geng, T. Zentgraf, L. Ju, F. Wang, and X. Zhang, vol. 474, no. 7349, pp. 64-67, 2011.

[2] B. Sensale-Rodriguez, T. Fang, R. Yan, M. M. Kelly, D. Jena, L. Liu, and H. Xing, Appl. Phys. Lett., vol. 99, no. 11, pp. 2009-2012, 2011.

[3] W. Gao, J. Shu, K. Reichel, D. V. Nickel, X. He, G. Shi, R. Vajtai, P. M. Ajayan, J. Kono, D. M. Mittleman, and Q. Xu, Nano Lett., vol. 14, no. 3, pp. 1242-1248, 2014.

[4]R. Yan, B. Sensale-Rodriguez, L. Liu, D. Jena, and H. G. Xing, Opt. Express, vol. 20, no. 27, pp. 28664-71, 2012.

[5] N. Kakenov, O. Balcı, E.O. Polat, H. Altan, C. Kocabas, J. Opt. Soc. Am., vol. 32, pp. 1861-1866,2015. 